\title{
Circulating microRNAs as potential non-invasive biomarkers in pediatric patients with celiac disease
}

\author{
${ }^{1}$ Medical molecular genetics Department, National Research Centre, Cairo, Egypt \\ ${ }^{2}$ Immunogenetics Department, National Research Centre, Cairo, Egypt; Microbiology and Immunology Department, \\ MSA University, Egypt \\ ${ }^{3}$ Immunogenetics Department, National Research Centre, Cairo, Egypt \\ ${ }^{4} \mathrm{Abu}$ El Reesh Children’s Hospital, Cairo University, Cairo, Egypt
}

\section{KEY WORDS \\ celiac disease; microRNA; gluten \\ free diet; biomarkers; auto- \\ antibodies}

\author{
Corresponding author \\ Eman Eissa \\ Immunogenetics department \\ National Research Centre \\ El Buhouth St., Dokki, \\ Giza Governorate \\ Cairo, Egypt \\ E-mail: emanessa82@yahoo.com
}

Doi

10.23822/EurAnnACI.1764-1489.90

\begin{abstract}
Summary
Celiac disease is an enteropathy induced by ingestion of gluten triggering an immune response in genetically predisposed individuals. MiRNAs are small non-coding RNAs that have a role as regulators of gene expression at the post transcriptional level. The aim of this study is to evaluate the possibility of using circulating miRNAs as non-invasive biomarkers in pediatric patients with celiac disease. In addition, we examine the effect of a gluten-free diet on the expression of these miRNAs in serum of CD patients. The expression pattern of miR-21 and miR-31 was estimated in serum of 25 untreated CD patients (recently diagnosed), 25 treated CD patients (on gluten-free diet) and 20 healthy controls using qRT-PCR. Our results demonstrated the significant up-regulation of microRNA-21 in the untreated celiac patients in comparison with the treated group and healthy controls. Moreover, miR-31 expression was significantly under-expressed in the untreated celiac patients in comparison with the treated group and healthy controls. Furthermore, the results showed that miR-21 expression level was significantly positively correlated with the tTG IgA auto-antibodies. In conclusion, circulating miRNA-21 and miRNA-31 could serve as potential non-invasive biomarkers for pediatric CD patients.
\end{abstract}

\section{Introduction}

Celiac disease $(C D)$ is an autoimmune disease affecting the small intestine with a prevalence of 1 in 100 to 1 in 200 subjects, particularly children (1-3). This enteropathy is triggered by the interaction of environmental and genetic factors $(3,4)$. $\mathrm{CD}$ is characterized by an immunological reaction against the TG2 (transglutaminase type 2) enzyme triggered by the ingestion of gluten peptides from wheat and related cereals in genetically predisposed individuals $(2,5,6)$. This autoimmune reaction induces a duodenal damage characterized by villous atrophy, intraepithelial lymphocytosis, infiltration of inflammatory cells in the lamina propria and crypt hyperplasia $(7,8)$. Defects in regulation of gene expression through microRNAs (miRNAs) could be responsible of the changes in intestinal permeability and intestinal immune system (2), suggesting their implication in the dysfunction of intestinal barrier and their association with certain clinical manifestation (9).

MiRNAs are small endogenous single-stranded non-coding RNAs that regulate gene expression through the control of stability and translation of the mRNA $(10,11)$. MiRNAs have been associated with various pathological conditions of the immune system (12). Many studies have reported the aberrant expression of miRNAs in intestinal biopsies of celiac patients, while the role of circulating miRNAs and their expression levels are still undefined compared to that of tissue miRNAs (4). Capuano et al. (2011) evaluated the miRNA expression pattern in the small intestine of children with active CD, children with CD on GFD and control children without CD. Their results showed the overexpression of miR-449a and the decrease of miR-124a expression in CD patients and GFD treated CD patients than in controls (13). 
In this study, we evaluate the possibility of using circulating miRNAs as non-invasive biomarkers in pediatric patients with celiac disease for diagnosis and prognosis. We investigate the deregulated expression pattern of miRNA-21 and miRNA-31 in serum of celiac disease patients. In addition, we examine the effect of a gluten-free diet on the expression of these miRNAs in serum of $\mathrm{CD}$ patients. We then analyze the correlation between these miRNA expression levels and the auto-antibodies of CD patients.

\section{Subjects and methods}

\section{Ethics}

This study was approved by the ethics committee of National Research Center, Giza, Egypt, and written informed consents were obtained from the parent/guardian of all children involved in our study before their enrollment.

\section{Study subjects}

This study included 70 subjects with age ranging from 2 to 14 years. They were divided into 3 groups: Group 1 consisted of 25 untreated CD patients (recently diagnosed); Group 2 consisted of 25 treated CD patients (on gluten-free diet) for about 5 years; Group 3 consisted of 20 healthy normal subjects matched for age and gender as a control group.

Patients were obtained from Cairo University Specialized Children Hospital, Cairo, Egypt. They were diagnosed according to the criteria of the European Society for Paediatric Gastroenterology Hepatology and Nutrition (analysis of autoantibodies
[anti-tTG and anti-endomysium IgA] by ELISA with investigation of intestinal biopsy) (14). Clinical manifestations and treatments of $\mathrm{CD}$ patients are summarized in table $\mathbf{I}$.

\section{RNA extraction and quantitative real-time PCR}

MicroRNA was extracted and isolated from plasma of all subjects of the study populations using miRNeasy Mini kit of Qiagen (Germany) according to the manufacturer's instructions. For miRNA-specific reverse transcription, microRNA was reverse-transcribed to cDNA using TaqMan ${ }^{\circledR}$ MicroRNA Reverse Transcription Kit (Applied Biosystems) and using specific primers according to the manufacturer's instructions. Reverse transcription was performed under the following conditions: 30 $\min$ at $16^{\circ} \mathrm{C}, 30 \mathrm{~min}$ at $42{ }^{\circ} \mathrm{C}$, and followed by $5 \mathrm{~min}$ at $85^{\circ} \mathrm{C}$, and the resulting cDNA was kept at $-80^{\circ} \mathrm{C}$ until use.

A real-time quantitative PCR (qRT-PCR) was carried out to quantify the expression levels in triplicate of mature miR31 and miR-21 using TaqMan ${ }^{\circledR}$ MicroRNA Assay kit and TaqMan ${ }^{\circledR}$ Universal Master Mix (Applied Biosystems) using step one real-time PCR system according to the manufacturer's instructions. RNU6B was used as endogenous control to normalize the expression levels of target miRs. Relative quantification (Rq) of miRNA expression was calculated using the $2-\Delta \Delta^{\mathrm{CT}}$ threshold cycle method. $\Delta \mathrm{Ct}$ was determined by subtracting the $\mathrm{Ct}$ values for RUN6B from the $\mathrm{Ct}$ values for the gene of interest. qRT-PCR was performed under the following conditions: $2 \mathrm{~min}$ at $50{ }^{\circ} \mathrm{C}, 10 \mathrm{~min}$ at $95^{\circ} \mathrm{C}$, followed by 50 cycles at $95^{\circ} \mathrm{C}$ for $15 \mathrm{~s}$, and at $60^{\circ} \mathrm{C}$ for $1 \mathrm{~min}$.

Table I - Clinical characteristics of the studied subjects.

\begin{tabular}{|c|c|c|c|}
\hline Characteristic & $\begin{array}{l}\text { Treated patients with celiac } \\
\text { disease }\end{array}$ & $\begin{array}{l}\text { Untreated patients with celiac } \\
\text { disease (recently diagnosed) }\end{array}$ & Normal healthy controls \\
\hline no. of cases & 25 & 25 & 20 \\
\hline age, (range) & $2-14$ & $2-14$ & $2-14$ \\
\hline disease duration, mean \pm sd (years) & $4.88 \pm 1.6$ & 0 & 0 \\
\hline $\begin{array}{l}\text { EMA IgA antibodies, no. positive/ } \\
\text { negative }\end{array}$ & $9 / 16$ & $15 / 10$ & $0 / 20$ \\
\hline medications (GFD) & $25 / 25$ & $0 / 25$ & $0 / 20$ \\
\hline biopsy & $\begin{array}{l}\text { villous shortening and relative } \\
\text { increase in intraepithelial } \\
\text { lymphocytes and mild chronic } \\
\text { duodenitis }\end{array}$ & $\begin{array}{l}\text { moderate enteritis with villous } \\
\text { atrophy and moderate duodenitis }\end{array}$ & - \\
\hline
\end{tabular}




\section{Statistical analysis}

Data were statistically analyzed using SPSS version 16.0 software (SPSS Inc., Chicago, Illinois, USA). Nonparametric T test (Kruskal-Wallis test) was used to compare gene expression levels between groups, and Spearman's rank correlation to test association between gene expression levels and auto-antibodies of patients. Data were presented as the mean \pm SEM. A $p$ value of less than 0.05 was considered statistically significant. Receiver operating characteristic (ROC) curve was constructed for each miRNA to evaluate the efficiency of miRNAs as biomarkers for CD patients against controls. Area under curve (AUC) values and 95\% confidence intervals for each miRNA were calculated.

\section{Results}

Deregulation of microRNA-21 expression pattern in plasma of $C D$ patients

This study demonstrated the significantly increased expression levels of microRNA-21 in the untreated celiac patients in comparison with the treated group and healthy controls (table II). The miR-21 expression was over-expressed in the treated CD patients compared with the control group (table II). In the untreated group, the expression of miR-21 was 8.49-fold increased compared to the control group (figure 1). In the treated group, miR-21 expression was 2.8-fold higher compared to normal controls (figure 1).

Deregulation of microRNA-31 expression pattern in plasma of $C D$ patients

Our results indicated that miR-31 expression was significantly down-regulated in the untreated celiac patients in comparison with the treated group and healthy controls and with insignificant down regulation in $\mathrm{CD}$ patients compared with the control group (table II). MiR-31 was 3-fold lower expressed in the untreated CD patients compared to the healthy controls, and 1.5-fold decreased compared to normal controls (figure 1).
Figure 1 - Fold changes of miR-21 and miR-31 expressions of the untreated and treated $C D$ patients were determined relative to normal controls. Bar graph represents the mean \pm SEM fold change.
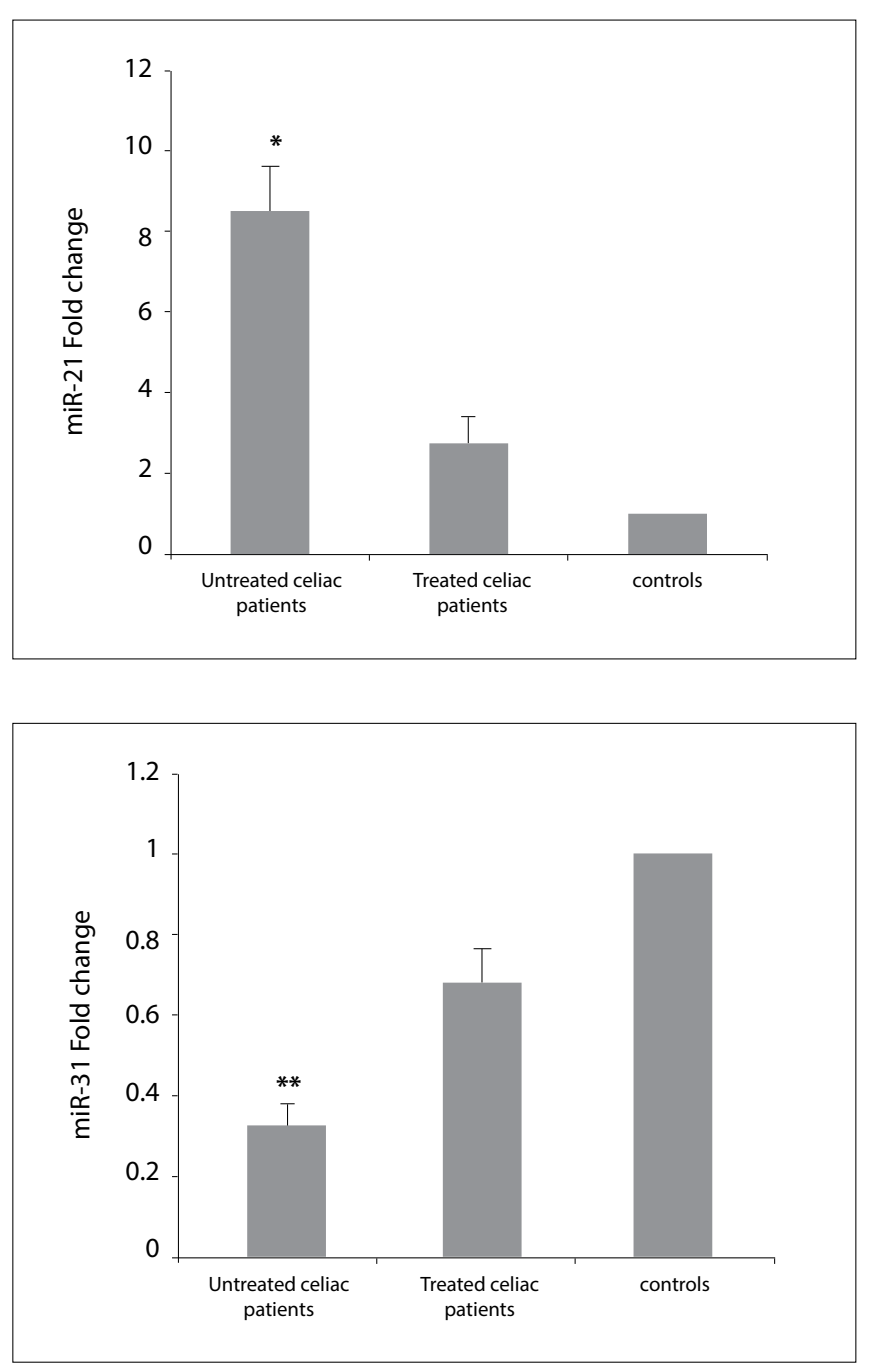

${ }^{1}$ Statistically significant at $\mathrm{p}<0.01$ versus controls (by Kruskal-Wallis test); ${ }^{2}$ Statistically significant at $\mathrm{p}<0.001$ versus controls (by Kruskal-Wallis test).

Table II - Expression levels of miR-31, miR-21 in plasma of untreated CD patients, treated CD patients, and normal healthy controls. The results were expressed as mean $\pm S E M$.

\begin{tabular}{llll}
\hline Parameters & $\begin{array}{l}\text { Untreated CD patients } \\
(\text { mean } \pm \text { SEM) }\end{array}$ & $\begin{array}{l}\text { Treated CD patients } \\
(\text { mean } \pm \text { SEM })\end{array}$ & $\begin{array}{l}\text { Normal controls } \\
(\text { mean } \pm \text { SEM })\end{array}$ \\
\hline miR-21 expression level & $135^{1} \pm 18$ & $44.3 \pm 10$ & $16 \pm 8$ \\
\hline miR-31 expression level & $0.45^{2} \pm 0.06$ & $0.9 \pm 0.13$ & $1.3 \pm 0.3$ \\
\hline${ }^{1}$ Statistically significant at $\mathrm{p}<0.001$ versus normal controls (Kruskal-Wallis test); ${ }^{2}$ Statistically significant at $\mathrm{p}<0.01$ versus normal controls (Kruskal-Wallis test).
\end{tabular}


Correlations between serum microRNAs and auto-antibodies in the untreated and treated CD patients

In the untreated and treated group, the results clarified that there is a significant positive correlation between miR-21 and tTG IgA auto-antibodies, while it has no correlation with EMA IgA auto-antibodies. Our data also showed no significant correlation between miR-31 expression levels with tTG IgA or EMA IgA auto-antibodies (table III).

\section{ROC curve of microRNA-21 and microRNA-31}

ROC curve showed that miR-21 has an AUC value of 0.847 (95\% CI $0.704-0.991)$, while miR-31 has an AUC value of $0.801(95 \%$ CI $0.658-0.944)$ at $\mathrm{p}<0.001$ versus normal controls. These findings revealed that both miR-21 and miR31 could function as good biomarkers for CD patients against healthy controls (figure 2, table IV).

\section{Discussion}

Specific miRNAs have been reported to be modulated in intestinal mucosa of $\mathrm{CD}$ patients, suggesting their involvement in the pathogenesis of $\mathrm{CD}$ and the probability of using thereof as diagnostic tools or as predictors of gluten free diet outcome in CD patients $(2,4)$.

Table III - Correlations between plasma miR-21 and miR-31 with auto-antibodies in the untreated and treated CD patients.

\begin{tabular}{|c|c|}
\hline \multicolumn{2}{|l|}{ Untreated CD patients } \\
\hline parameters & $\begin{array}{l}\mathrm{R} \text { (Spearman } \\
\text { correlation) }\end{array}$ \\
\hline miR-21 expression - tTG IgA auto-antibodies & $0.661^{1}$ \\
\hline miR-21 expression - EMA IgA auto-antibodies & -0.123 \\
\hline 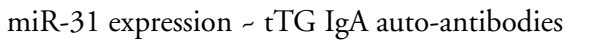 & -0.257 \\
\hline miR-31 expression - EMA IgA auto-antibodies & 0.112 \\
\hline \multicolumn{2}{|l|}{ Treated CD patients } \\
\hline parameters & $\begin{array}{l}\mathrm{R} \text { (Spearman } \\
\text { correlation) }\end{array}$ \\
\hline miR-21 expression - tTG IgA auto-antibodies & $0.775^{2}$ \\
\hline miR-21 expression - EMA IgA auto-antibodies & -0.160 \\
\hline miR-31 expression - tTG IgA auto-antibodies & 0.291 \\
\hline miR-31 expression - EMA IgA auto-antibodies & -0.341 \\
\hline
\end{tabular}

${ }^{1}$ Correlation is significant at the 0.01 level (2-tailed); ${ }^{2}$ Correlation is significant at the 0.001 level (2-tailed).
Figure $2-R O C$ curve of miR-21 and miR-31 for patients with celiac disease versus healthy controls.

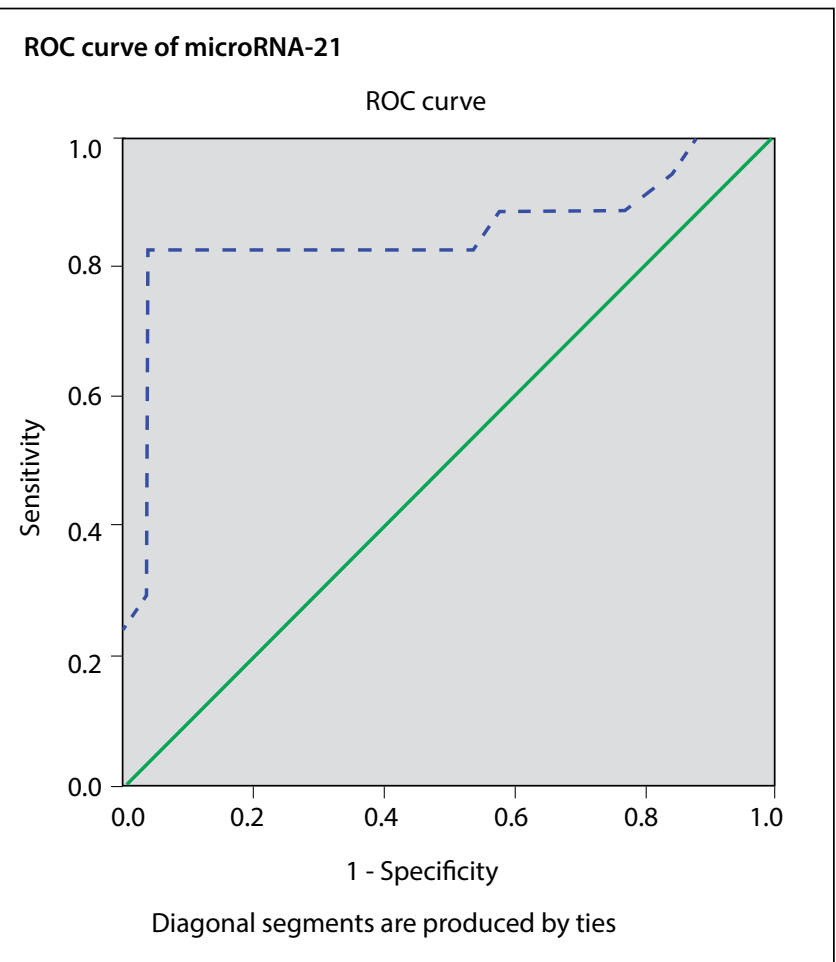

ROC curve of microRNA-31

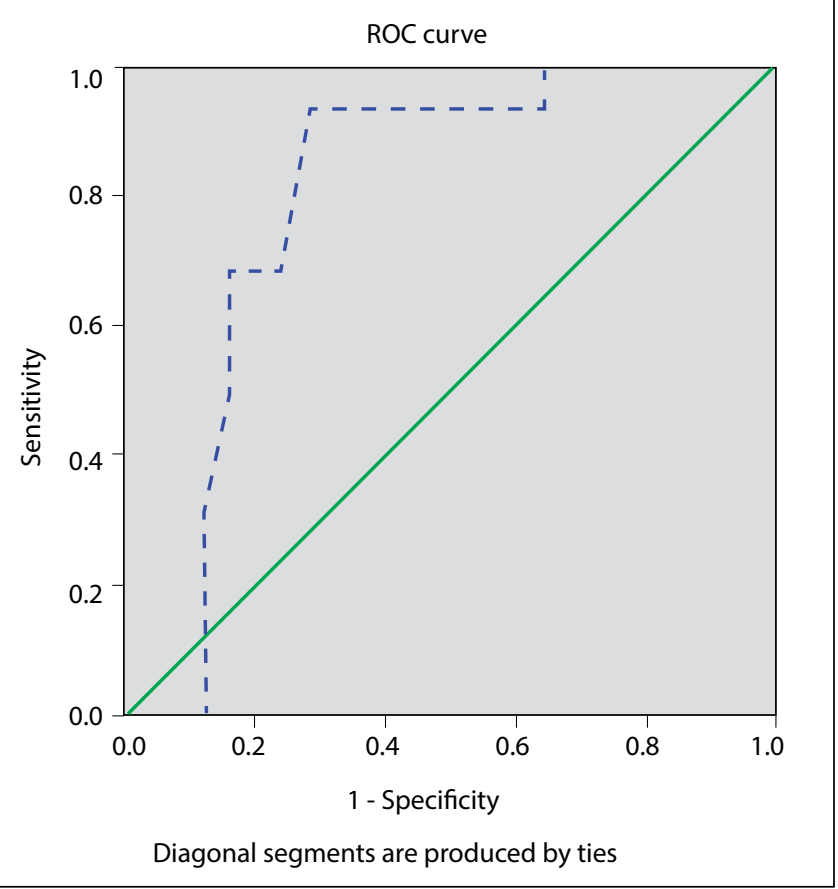


Table IV - AUCs and 95\% Confidence Intervals of miRNA-31 and miRNA-21 for CD patients versus normal controls.

\begin{tabular}{lcccccccc}
\hline & \multicolumn{7}{c}{ CD patients against normal controls } \\
\cline { 2 - 9 } & AUC & $\begin{array}{c}\text { standard } \\
\text { error }^{2}\end{array}$ & $\begin{array}{c}\text { 95\% confidence } \\
\text { intervals }\end{array}$ & sensitivity & specificity & LR+ & $\begin{array}{c}\text { LR- } \\
\text { val-off } \\
\text { value }\end{array}$ \\
\hline miRNA-21 & $0.847^{1}$ & 0.073 & $0.704-0.991$ & $82.4 \%$ & $80.8 \%$ & 4.3 & 0.22 & 61.28 \\
\hline
\end{tabular}

${ }^{1}$ Statistically significant at $\mathrm{p}<0.001$ versus controls; ${ }^{2}$ Under the nonparametric assumption.

Our study demonstrated that miR-21 expression was significantly up-regulated in active $\mathrm{CD}$ patients compared to healthy controls and also children with CD on a gluten-free diet. In addition, the expression level of miR-21 was decreased in the treated CD patients compared to untreated patients. Moreover, in the treated and untreated CD patients, the results showed that miR-21 expression level was significantly positively correlated with the tTG IgA auto-antibodies, while there was no correlation between miR-21 and EMA IgA auto-antibodies. These findings are in line with results of Buoli Comani et al. (2015), who found a significant over-expression of miR-21-5p expression in the duodenal biopsies of active CD patients in comparison with controls. In addition, $\mathrm{CD}$ patients on a gluten-free diet showed a decrease in miR-21-5p compared to controls with a non-significant difference in expression patterns. MiR-21 is also widely reported as dysregulated in UC and CD (15). Ludwig et al. (2013) found that miR-21 was upregulated in IBD-associated dysplastic lesions compared with active patients with IBD (16). In a study of intestinal miRNA levels in CD, Wu et al (2010) identified several miRNAs that are upregulated (miR16, -20a, -21, and -106a) (17). Let-7b, miR16, and miR-21 were greatly expressed in human dendritic cells, which likely contribute to the chronic inflammation of CD $(18,19)$.

There were many studies reported that miR-21 had a proinflammatory role in IBD by impairing intestinal barrier function. Paraskevi et al. (2012) and Yang et al. (2013) found up-regulation of miR-21 in patients with UC in both the mucosal and blood samples $(20,21)$. MiR-21 was found to affect the intestinal epithelial permeability by targeting RhoB, which was found significantly decreased in the patients with UC. In addition, intestinal integrity and morphology were declined in Caco-2 cells and in UC patients exhibiting overexpression of miR-21 through targeting RhoB (21).

Similarly, in IBD patients Shi et al. (2013) demonstrated the up-regulation of miR-21. Furthermore, their results showed the increase of intestinal permeability and epithelial cell apoptosis promoted by dextran sulphate sodium were attenuated in miR21 knockout mice (22).

On the other hand, our study results showed that miR-31 expression was significantly under-expressed in the untreated ce- liac patients in comparison with the treated group and healthy controls. Moreover, its expression was restored in the treated $\mathrm{CD}$ patients compared to the untreated celiac patients. In addition, miR-31 expression level was not correlated with tTG IgA nor EMA IgA auto-antibodies in both the untreated CD group and treated $\mathrm{CD}$ group.

These results are similar to that of previous studies in which, Vaira et al. (2014) investigated the duodenal mucosa miRNA expression profile and confirmed significant deregulation of miR-31-5p and miR-551b-5p in classical CD patients, and five miRNAs in anaemic CD patients (miR-31-5p, miR-192-3p, miR-551b-5p, miR-638 and miR-1290) compared with non$\mathrm{CD}$ controls (6). In addition, the duodenal fibroblasts obtained from patients are then incubated with gliadin peptides (13 and $33 \mathrm{mer}$ ) and measured for the miRNA expression. The deregulation of miRNA levels was observed in untreated $C D$ patients for miR-192-3p, miR-31-5p and miR-1285-3p (6).

Buoli Comani et al. (2015) analyzed duodenal biopsies of pediatric celiac patients for examining a panel of miRNAs and their target genes compared to controls. After that, they evaluated the circulating miRNA patterns in untreated $C D$ patients or on a gluten-free diet compared to controls. They found that miR$31-5 p$ and miR-338-3p were underexpressed in the duodenum samples of CD patients (2). In addition, circulating miR-31-5p in untreated $C D$ patients displayed a significantly decline compared to controls. Moreover, there is no statistically significant difference in miRNA levels between controls and CD patients on a gluten-free diet (2).

In a study performed by Magni et al. (2014) in the duodenum of adult CD patients, the significantly decreased expression of miR-192-5p, miR-31-5p, miR-338-3p, and miR-197 were demonstrated as compared with controls (5). FOXP3, the target of miR-31-5p, showed upregulation in CD patients. Furthermore, exposure of $\mathrm{CD}$ patients to gliadin led to changes in the expression of FOXP3, miR-192-5p, miR-31-5p, CXCL2 and NOD2 (5).

Olaru et al. (2011) found that miR-31 was increased successively at each stage of IBD progression from non-inflamed to inflamed non-neoplastic, dysplastic, and finally cancer (23). In addition, RT-PCR analysis revealed altered expression of miR- 
$31,-125 a,-142-3 p$, and $-146 a$ discriminating between the inflamed mucosa of CD and UC (24).

In our previous study, we showed the aberrant expression of miR-31 and miR-21 in SLE patients compared to their first-degree relatives and controls. Moreover, our results indicated that both of miR-31 and miR-21 could serve as regulatory biomarkers in patients with SLE (25). This may indicate the altered expression of these circulating miRs in the autoimmune diseases.

\section{Conclusion}

Our study demonstrated the deregulation of circulating miRNA-21 and miRNA-31 expression levels in children with CD and showed that miR-21 expression level was positively correlated with the tTG IgA auto-antibodies. In addition, our findings indicated that a gluten free diet has influenced the expressions of miRNA-21 and miRNA-31 in serum of pediatric CD patients. Therefore, circulating miRNA-21 and miRNA-31 could be used as potential non-invasive diagnostic and prognostic biomarkers for pediatric $\mathrm{CD}$ patients.

\section{Funding}

This study was funded by National Research Centre, Cairo, Egypt.

\section{Conflict of Interest}

The authors declare that they have no conflict of interest.

\section{References}

1. Kenrick K, Day AS. Coeliac disease: Where are we in 2014? Aust Fam Physician 2014; 43(10):674-678.

2. Buoli Comani G, Panceri R, Dinelli M, et al. MiRNA-regulated gene expression differs in celiac disease patients according to the age of presentation. Genes Nutr 2015; 10(5):482.

3. Lebwohl B, Sanders DS, Green PHR. Coeliac disease. Lancet 2018; 391(10115):70-81.

4. Felli C, Baldassarre A, Masotti A. Intestinal and Circulating MicroRNAs in Coeliac Disease. Int J Mol Sci 2017; 18(9). pii: E1907.

5. Magni S, Buoli Comani G, Elli L, et al. MiRNAs affect the expression of innate and adaptive immunity proteins in celiac disease. Am J Gastroenterol 2014; 109(10):1662-1674.

6. Vaira V, Roncoroni L, Barisani D, et al. MicroRNA profiles in coeliac patients distinguish different clinical phenotypes and are modulated by gliadin peptides in primary duodenal fibroblasts. Clin Sci (Lond) 2014; 126(6):417-423.

7. Elli L, Bergamini CM, Bardella MT, et al. Transglutaminases in inflammation and fibrosis of the gastrointestinal tract and the liver. Dig Liver Dis 2009; 41(8):541-550.

8. Bascuñán-Gamboa KA, Araya-Quezada M, Pérez-Bravo F. MicroRNAs: An epigenetic tool to study celiac disease. Rev Esp Enferm Dig 2014; 106(5):325-333.
9. Zhang L, Cheng J, Fan XM. MicroRNAs: New therapeutic targets for intestinal barrier dysfunction. World J Gastroenterol 2014; 20(19):5818-5825.

10. Esteller M. Non-coding RNAs in human disease. Nat Rev Genet 2011; 12 (12):861-874.

11. Guo H, Ingolia NT, Weissman JS, et al. Mammalian micro-RNAs predominantly act to decrease target mRNA levels. Nature 2010; 466(7308):835-840.

12. Dai R, Ahmed SA. MicroRNA, a new paradigm for understanding immunoregulation, inflammation, and autoimmune diseases. Transl Res 2011; 157(4):163-179.

13. Capuano M, Iaffaldano L, Tinto N, et al. MicroRNA-449a overexpression, reduced NOTCH1 signals and scarce goblet cells characterize the small intestine of celiac patients. PLoS One 2011; 6 (12): e29094.

14. Husby S, Koletzko S, Korponay-Szabó IR, et al.; ESPGHAN Working Group on Coeliac Disease Diagnosis; ESPGHAN Gastroenterology Committee; European Society for Pediatric Gastroenterology, Hepatology, and Nutrition. European society for pediatric gastroenterology, hepatology, and nutrition guidelines for the diagnosis of coeliac disease. J Pediatr Gastroenterol Nutr 2012; 54(1):136-160.

15. Peck BC, Weiser M, Lee SE, et al. MicroRNAs Classify Different Disease Behavior Phenotypes of Crohn's Disease and May Have Prognostic Utility. Inflamm Bowel Dis 2015; 21(9):2178-2187.

16. Ludwig K, Fassan M, Mescoli C, et al. PDCD4/miR-21 dysregulation in inflammatory bowel disease-associated carcinogenesis. Virchows Arch 2013; 462(1):57-63.

17. Wu F, Zhang S, Dassopoulos T, et al. Identification of microRNAs associated with ileal and colonic Crohn's disease. Inflamm Bowel Dis 2010; 16(10):1729-1738.

18. Silva MA, López CB, Riverin F, et al. Characterization and distribution of colonic dendritic cells in Crohn's disease. Inflamm Bowel Dis 2004; 10(5):504-512.

19. Cekaite L, Clancy T, Sioud M. Increased miR-21 expression during human monocyte differentiation into DCs. Front Biosci (Elite Ed) 2010; 2(3):818-828.

20. Paraskevi A, Theodoropoulos G, Papaconstantinou I, et al. Circulating MicroRNA in inflammatory bowel disease. J Crohns Colitis 2012; 6(9):900-904.

21. Yang Y, Ma Y, Shi C, et al. Overexpression of miR-21 in patients with ulcerative colitis impairs intestinal epithelial barrier function through targeting the Rho GTPase RhoB. Biochem Biophys Res Commun 2013; 434(4):746-752.

22. Shi C, Liang Y, Yang J, et al. MicroRNA-21 knockout improve the survival rate in DSS induced fatal colitis through protecting against inflammation and tissue injury. PLoS One 2013; 8(6):e66814.

23. Olaru AV, Selaru FM, Mori Y, et al. Dynamic changes in the expression of microRNA-31 during inflammatory bowel disease-associated neoplastic transformation. Inflamm Bowel Dis 2011; 17(1):221-231.

24. Béres NJ, Kiss Z, Sztupinszki Z, et al. Altered mucosal expression of microRNAs in pediatric patients with inflammatory bowel disease. Dig Liver Dis 2017; 49(4):378-387.

25. Amr KS, Bayoumi FS, Elgengehy FT, et al. The role of microRNA-31 and microRNA-21 as regulatory biomarkers in the activation of T lymphocytes of Egyptian lupus patients. Rheumatol Int 2016; 36(11):1617-1625. 